\title{
Interactive comment on "Short communication: Multiscalar drag decomposition in fluvial systems using a transform-roughness correlation (TRC) approach" by David L. Adams and Andrea Zampiron
}

Anonymous Referee \#1

Received and published: 30 July 2020

The manuscript entitled "Multiscalar drag decomposition in fluvial systems using a transform-roughness correlation (TRC) approach", submitted for publication as a short communication to Earth Surface Dynamics presents an interesting study regarding the determination of flow resistance in fluvial channels. The presented method is based on a the thalweg profile and good results are presented by the authors. Although the topic of the manuscript fits the scope of the journal, I found that it is not suitable for publication in its present form due to various reasons. For example, the authors need to expand their consideration towards the heterogeneity of the surface (and not only a 
single profile). In fact, focusing solely on the thalweg profile means that some morphological features such as banks etc. are not adequately captured through the analysis. This is, however, only one major issue that I identified, as becomes apparent from my comments below, in which I also comment on the experimental data, experimental procedure, theoretical background, data analysis, data interpretation, and the number of references. Overall, the approach is certainly interesting, but it cannot be presented in the form of a short communication (in my opinion), as there are simply too many open questions and too many shortcuts in the manuscript. I therefore recommend rejection of the manuscript and resubmission as a research paper.

Detailed comments: L11: Having read the communication, I am not sure what conventional equation is meant by the authors. L22: It is stated that the grains and bedforms on the surface span orders of magnitude of scales. So the question arises if the thalweg profile is really sufficient to capture all spatial scales? (see also L48) L50: The reference for the used roughness correlation should be given here, as it was not really developed by the authors. L62: There are many morphological features that contribute to drag which are not considered when analyzing the thalweg profile (this includes the curvature of the channel, alternate bars and many more). This needs to be highlighted better. L64: It is stated that the spatial extent of the data should at least cover the largest features that produce significant drag (resistance). A dune is one such feature and it becomes not really clear why many dune crests need to be included in the dataset. This should be elaborated in some more depth. In this context, can the spatial extent of 3D-features really be described using a single profile? L66: The data meant by the authors should be specified, as I am not sure how data can be reduced to streamlines. A streamline is a line that follows the direction of flow velocity and is a hydraulic feature. So how exactly can topographic data be reduced to a streamline? Also, how can streamlines intersect when these lines follow the direction of flow? This should be specified. L70: I fully acknowledge that the authors use wavelet approach. However, the principle of wavelets should be described in some more depth, as not all reader will be familiar with the principles of the wavelet transforms described here.

\section{ESurfD}

Interactive comment
Printer-friendly version

Discussion paper 
L76: Fig. 1 presents data from Experiment 1a - these data have not been described and no reference is found to an experimental study. This is confusing. For example, how does the reader know the shown profile originates from a riffle-bar sequence and that the MODWT is aligned with the thalweg elevation profile? In this context, why is the CWT not aligned with the thalweg-profile? It is also a wavelet transform that is based on the thalweg-profile - so it should be aligned with this profile? This could be formulated more clearly. L84: I fully agree but would also argue that almost all gravel bed rivers are in the hydraulic rough regime. In this context, what is meant by the statement that roughness correlations have only been developed for limited ranges of $R^{*}$ ? Typically, three ranges are distinguished, and existing approaches can be found that cover all these ranges. L85: I am not sure that the width to depth ratio is an appropriate measure to determine if the flow is $2 \mathrm{D}$. For example, the flow in the roughness layer of gravel beds is far from being 2D. Note also 2D conditions also depend on the relative submergence ( $\mathrm{I}$ acknowledge that this is mentioned in the next paragraph, but I would expect such a statement already here). L95: In this case I could also agree with the 2D-statement from the last paragraph. L96: The TRC approach remains a black box until here. L100: How was discharge measured? L101: How was sediment transport scaled? L104: What was the working principle of these gages and what was the distance between them? Investigating Table 2, I found that the water depths were very low $(<0.02 \mathrm{~m})$. This means that surface tension can impose a significant scaleeffect biasing the results. This needs to be discussed. In this context, what was the accuracy of the water depth readings? I am asking because the differences in water depth are within the millimeter range. L111: How exactly can discharge be scaled with the width of the experimental channel when using a Froude-scale model? The information that bankfull discharge was used is enough, but it should be stated how deep the channel was. Also, is there information available how much discharge was conveyed through the sediment bed (this could be important given the low discharges)? L116: Which weirs? Was there a backwater effect? L117: What was the corresponding flow rate? That would help to answer my question @L111? L118: Had the draining of the

\section{ESurfD}

Interactive comment
Printer-friendly version

Discussion paper 
bed any effect on the topography and was the bed saturated again when increasing the discharge after the measurements? Table 2 lacks of units and $k^{*}$ s,pred has not been defined properly (see below). L121: Referring back to my comment @L104 it is stated 1hat the water surface elevation was determined to the nearest millimeter and that the mean water depths are lower than the maximum water depth (what is meant by the statement "most of the maximum flow depths"?), the degree of precision is different than stated @L122. L141: Figure 3 shows the thalweg for the total length of the table of Exp1a and the corresponding profile is shown in Fig.1a. This means to me that there is an effect of the inflow and outflow section on the bed morphology (which is directly visible in Fig. 1a) which in turn may affect the results of the wavelet analysis. This needs to be evaluated. There is also a mismatch between the thalweg elevations the figures - please explain. Moreover, the thalweg is also meandering how was this accounted for? Note also that it was mentioned that the bricks represent a linear reference elevation. Looking at the colors of the bricks and the color-scale of Fig. 3 I would actually disagree with this statement (note also that no units are given for the scale). Another comment concerns the wetted width which, according to the numbers presented in Table 2, was different, even for bankfull conditions. I calculated the wetted width for $\operatorname{Exp} 1 \mathrm{c}(3)$ from the numbers presented in Table2 and the formulas given at L143 and 144 and obtained a wetted width of $0.32 \mathrm{~m}$ which is larger than the channel width of $0.30 \mathrm{~m}$. How is that possible? This in turn raises some serious questions regarding the accuracy of the experimental data which needs to be discussed in much more depth given the small scale of the experiments (see also my comment regarding the surface tension). This, together with the influence of the inlet and outlet sections indicates that more work is required to substantiate the results of the study. L149: What was the range of the discharges and what was the grain-size distribution of the bed material? L157: Strictly speaking, drag is not estimated. What is estimated is k_s, a roughness length. L159: Since skewness plays an important role, it would be good to show corresponding distributions. L166: What exactly is meant by "diversity of roughness peak heights"? This remains unclear. L174: I see five peaks in the profile in
ESurfD

Interactive comment
Printer-friendly version

Discussion paper 
Fig.1 - this number is not sufficient? (I acknowledge my comment above regarding the influence of the inflow and outflow section). Nonetheless, this needs to be discussed in more detail. L175: What was the range of delta in the present investigation? L178: I don't understand Figure 7 from the previous presentation of the material. L179: But k_s is a length scale which is different from drag. Please clarify. L183 and following: See my comment regarding the influence of the in- and outlet sections on the morphology of the bed. L190: I assume that the profile was detrended for the analysis, i.e. the bed slope is not considered in this analysis? L197: What is meant by $k$ s,pred? How was this determined? L201: It is stated that topographic variation tends towards zero, but above (L191) it is stated that the effective slope is greatest at grain-scale wave lengths (this could also be seen as a measure of topographic variation at another scale). This is contradictory in my opinion. Please explain. L024: This figure is the same as the Form Size Distribution proposed by Nyander et al (2003)? How is it possible that it is the same? Please be more specific. L205: I am confused now. Here it is stated that Equation 1 is used to predict $k \_s$, which is ok. But what is then the relative value of k_s,pred (see L 197)? I also see the need to define the concept of determining $k$ s for different wavelengths from a hydraulic point of view in more detail taking the physics into account (and the assumptions on which the determination of $k \_s$ is based: For example, were local values of velocities and slopes used? How was it ensured that uniform flow conditions prevailed? What about 2D-flow conditions at the grain scale? Don't get me wrong, the presented results are certainly interesting, but this needs to be elaborated in much more depth in my opinion. L213: The DEM is not analyzed, but the thalweg-profile extracted from the DEM. L214: The mother wavelet is only mentioned in the figure caption indicating the need to present the chosen approach in much more detail. This is rather confusing (also the choice of the other wavelets indicated in Figure 7 which remains a black box to me). L218: I would argue that I only see one extreme outlier. L224: I basically agree, and this would be one step towards answering my comment @L205. However, I am not sure that I understand the statement regarding the proportionality - it should give the same value. L226: I still do not understand ex-

\section{ESurfD}

Interactive comment
Printer-friendly version

Discussion paper 
actly how $\mathrm{k}^{*}$ s,pred was obtained. What is meant without the transform? Does that mean the overall profile was used? This needs to be described in much more depth.

ESurfD L229: Why does that validate the TRC approach? The numbers deviate (see my comment @L224). A comment in between - all this compares (to my understanding) the presents results in regard to the approach of Forooghi et al. (2017) - but how does that approach relate to the real k_s value? That means what is the "real" $k \_s$ value from the experiments? This needs to be discussed in depth. It seems that the hydraulic data have not been used to determine k_s (I might be wrong here, but this indicates that a more precise presentation of the material is required). Figure 9: First, see my comments regarding the experimental data. Second, why is $k^{*} \_$,pred used here and not sum(k_s,pred). This is confusing, as the latter parameter has been derived but is not presented in this final plot. L240 and following: Please consider my above comments. L251 and following: This main information here should have been presented in the introduction in my opinion. L268 and following: Please consider my above comments. References: The short communication is overloaded with references.

Interactive comment on Earth Surf. Dynam. Discuss., https://doi.org/10.5194/esurf-2020-51, 2020.
Interactive comment 